\title{
Effects of mechanical and electrical coupling on the parametric sensitivity of mode localized sensors
}

\author{
P. Thiruvenkatanathan, J. Yan, J. Woodhouse, A. Aziz and A. A. Seshia \\ Department of Engineering, Trumpington street, \\ University of Cambridge, \\ Cambridge, United Kingdom
}

\begin{abstract}
We compare and contrast the effects of two distinctly different mechanisms of coupling (mechanical and electrical) on the parametric sensitivity of micromechanical sensors utilizing mode localization for sensor applications. For the first time, the strong correlation between mode localization and the phenomenon of 'eigenvalue loci-veering' is exploited for accurate quantification of the strength of internal coupling in mode localized sensors. The effects of capacitive coupling-spring tuning on the parametric sensitivity of electrically coupled resonators utilizing this sensing paradigm is also investigated and a mass sensor with sensitivity tunable by over $400 \%$ is realized.
\end{abstract}

Keywords-Mode localization, Eigenvalue curve veering, microelectromechanical resonant sensors, Sensitivity enhancement

\section{INTRODUCTION}

Vibration localization has been employed as an ultrasensitive approach to detecting small perturbations in the structural properties of micromechanical sensors [1-3]. Besides its high sensitivity to structural perturbations (mass/stiffness), an eigenstate shift based sensor offers the added advantage of intrinsic common mode rejection that makes it less susceptible to false positive outputs arising from ambient environmental fluctuations [2], [4]. Parametric sensitivities that are orders of magnitude greater than the more conventionally used resonant frequency shift approach have been experimentally demonstrated using this sensing paradigm [1-3]. Further enhancements in the sensitivity in such sensors however, depend critically on the ability to control and predict the strength of the internal coupling between the resonators. In this paper, we compare the effects of two disparate mechanisms of coupling - mechanical and electrical coupling, on the parametric sensitivity of micromechanical sensors utilizing this paradigm of sensing. Furthermore, in an attempt to improve the accuracy of such sensors, we pay special attention to the behaviour of the eigenvalues of the system when localization occurs and exploit the associated phenomenon of eigenvalue curve veering for the accurate experimental quantification of the internal coupling spring constants between the coupled resonators. We also explore the potential for tuning parametric sensitivity in mode-localized sensors that are electrically coupled and realize a mass sensor with a parametric sensitivity tunable by over $400 \%$ by simply altering the applied DC bias voltages.

\section{MODE LOCALIZATION AND CURVE VEERING}

In an array of identical resonators coupled through weak springs, a small perturbation in the structural properties of one of the resonators strongly impacts coupled oscillations causing the vibration modes to localize. The extent of this vibration energy confinement depends not only on the magnitude of the induced disorder, but also on the strength of internal coupling between the resonators with weaker coupling resulting in stronger localization of the vibration modes. Mode localization is also associated with the phenomenon of 'eigenvalue curve veering' [5-7]. Curve veering or loci veering refers to the abrupt divergence of the eigenvalues of coupled oscillators as they approach each other when a system parameter is varied [7]. All of the modal properties get interchanged at this point of transition or veering of the eigenvalues [5]. It has been shown that the existence of close eigenvalues in a coupled vibratory system is likely to cause the occurrence of both curve veering and mode localization suggesting that they are both manifestations of the same drastic phenomenon [6]. In order to understand the physics underlying the phenomena, let us consider a simple system of two coupled oscillators as shown in Fig. 1.

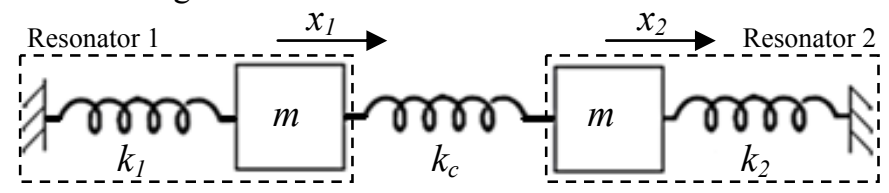

Figure 1. Discrete element model of two coupled oscillators.

In Fig. 1, ' $m$ ' represents the mass of resonators 1 and 2 $\left(m_{1}=m_{2}=m\right) ; k_{1}$ and $k_{2}\left(=k_{1}+\Delta k\right)$ represent their respective stiffnesses; $k_{c}$ - the internal coupling spring constant; $x_{1}, x_{2}$ their displacements. The two important parameters that we pay special attention to are the non-dimensionalized coupling factor $\left(\kappa=k_{c} / k_{1}\right)$ between the two oscillators and the nondimensionalized stiffness disorder on resonator 2 relative to resonator $1 \quad\left(\delta=\Delta k / k_{1}\right)$. The eigenvalue problem for undamped free vibration may be expressed as-

$$
\left|\begin{array}{cc}
\beta_{1}-\lambda & -\kappa \\
-\kappa & \beta_{2}-\lambda
\end{array}\right|=0
$$

where $\beta_{1}=1+\kappa ; \beta_{2}=1+\kappa+\delta$ and $\lambda=\omega_{n}{ }^{2} / k_{1} / m\left(\omega_{n}\right.$ represents the angular resonant frequencies of vibration for the two modes $n=1 ; 2$ ). The corresponding eigenvalues are -

$$
\lambda=\frac{1}{2}\left\{\beta_{1}+\beta_{2} \pm \sqrt{\left(\beta_{1}-\beta_{2}\right)^{2}+4 \kappa^{2}}\right\}
$$


with eigenvector components given by:

$$
\frac{x_{2}}{x_{1}}=\frac{1+\kappa-\lambda}{\kappa} .
$$

When the disorder in the stiffness of resonator 2 is zero, i.e., when $\delta=0 ;\left(\beta_{1}=\beta_{2}\right)$, the system is tuned or ordered and the eigenvalues and the corresponding eigenstates of the system may be expressed as -

$$
\lambda_{01}=1, \frac{x_{2}}{x_{1}}=+1 ; \lambda_{02}=1+2 \kappa, \frac{x_{2}}{x_{1}}=-1
$$

Under conditions of weak internal coupling $(\kappa<<1)$, plotting the loci of the two eigenvalues of the system $(\lambda)$ versus the disorder in the stiffness of resonator $2(\delta)$ yields Fig. 2. It may be observed that the loci of the two eigenvalues do not cross but rather veer abruptly with high local curvatures as the two eigenvalues approach each other. The point of transition corresponds to the point at which the system is symmetric $\left(\beta_{1}=\beta_{2}\right)$ as illustrated in Fig. 2 and represented in (4). At values where $\left(\beta_{1}-\beta_{2}\right)^{2} \gg\left(4 \kappa^{2}\right)$, however, the modes localize as seen from (3) and (2) and illustrated in Fig. 2.

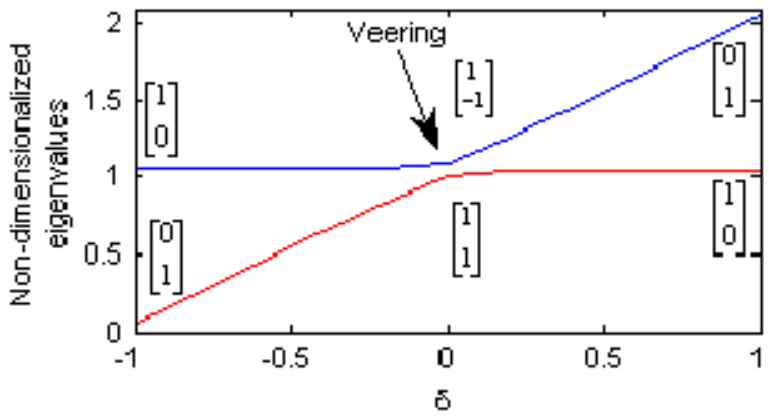

Figure 2. Loci of the dimensionless eigenvalues of the two coupled oscillators in terms of the disorder $\delta$.

Since the eigenvalues at the transition/veering zone correspond directly to the point at which the system is symmetric, (4) may be utilized to accurately quantify the strength of internal coupling between the resonators if the resonators could be tuned to the transition region. It is to be noted that weaker coupling springs result in higher local curvatures at the veering zone, suggesting a more rapid transition of the eigenfunctions even for small symmetry breaking perturbations in stiffness - consequently causing the mistuned system to become strongly localized. Furthermore, the closeness of the eigenvalues is also dictated by the strength of internal coupling between the resonators with weaker coupling spring constants resulting in closer eigenvalues as illustrated in Fig. 3. While the analytical description so far has been with the assumption of a positive coupling spring constant $\left(k_{c}\right)$ between the coupled oscillators i.e., a mechanically coupled resonator configuration, the presence of a negative coupling spring as in the case of electrical coupling, inverts the phase performance and in consequence, the modal behaviour of the system at the two eigenvalues. Nevertheless, the phenomenon of frequency veering would still exist under such conditions as the eigenfunctions are now simply interchanged at the two eigenvalues.

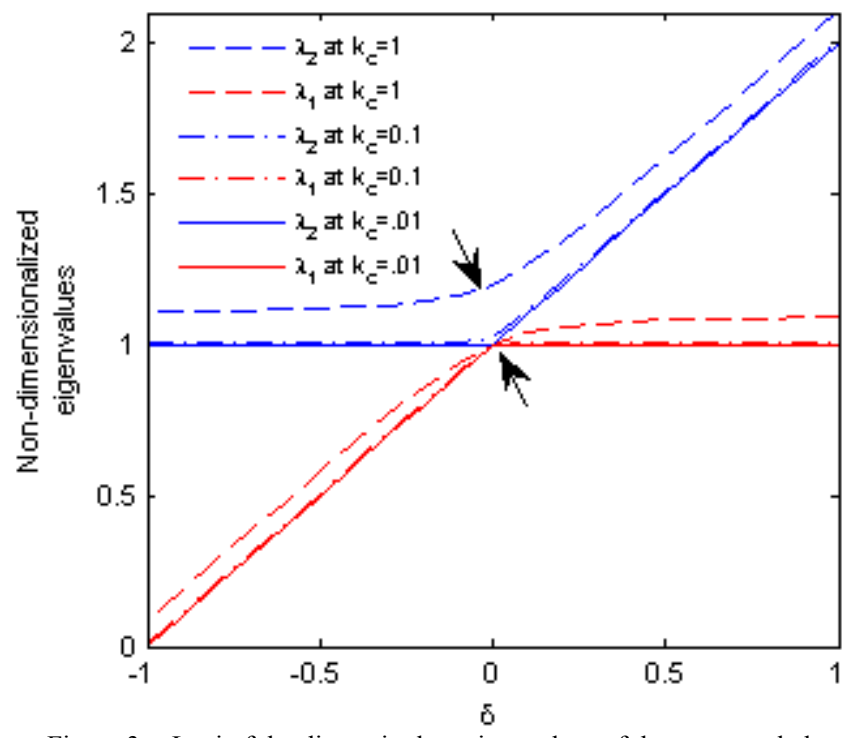

Figure 3. Loci of the dimensionless eigenvalues of the two coupled oscillators in terms of the disorder $\delta$ at varying strengths of internal coupling.

Utilizing curve veering for accurate quantification of the internal coupling spring constant becomes especially important when utilizing weakly coupled oscillators for mode-localized sensing applications. This is due to the critical dependence of the measured relative shifts in the eigenstates on the strength of internal coupling between the coupled oscillators. The relative shifts in the eigenstates due to small perturbations in mass or stiffness in a pair of weakly coupled oscillators may be expressed as in (5) and (6) respectively [3], [1]:

$$
\begin{aligned}
& \left|\frac{\Delta u_{i}}{u_{0 i}}\right| \approx\left(\frac{1}{4}+\frac{1}{4\left(k_{c} / k\right)}\right) \frac{\Delta m}{m} ;(i=1,2) \\
& \left|\frac{\Delta u_{i}}{u_{0 i}}\right| \approx \frac{\Delta k}{4 k_{c}} ;(i=1,2)
\end{aligned}
$$

It is clear from (5) and (6) that any inaccuracy in the quantification of the scaled coupling factor directly results in errors in the calculated eigenmode shift in such a sensing paradigm. Furthermore, this approach is even more useful for mechanically coupled mode localized sensors as experimental quantification of the coupling factor in resonators subjected to weak mechanical coupling is not as straightforward as in the electrical case. Moreover, fabrication tolerances in the micromachining process often limit perfect matching of the coupled resonators and hence quantifying the strength of internal coupling from the observed resonant frequency spectrum without performing the veering test would result in an uncalibrated output. In what follows, we utilize the electrical spring softening effect to induce parametric variations in the stiffness between coupled micromechanical resonators, and plot the eigenvalue loci of the system until the loci veer. We then use the point of veering to deduce the scaled coupling spring constant and utilize the value thus deduced, to accurately predict the relative shifts in the eigenstates to small, induced symmetry breaking perturbations in mass, and thus, use the system as a highly sensitive mass sensor. Results from the relative shifts in the eigenstates deduced with and without performing the initial eigenvalue curve veering test are 
compared and an improvement in accuracy of over $200 \%$ is demonstrated.

\section{IMPLEMENTATION AND VERIFICATION}

\section{A. Device descriptions}

The concept is experimentally demonstrated here using two pairs of double ended tuning fork (DETF) resonators subjected to electrical and mechanical coupling - scanning electron microscope (SEM) images of which are shown in Figs. 4 and 5.
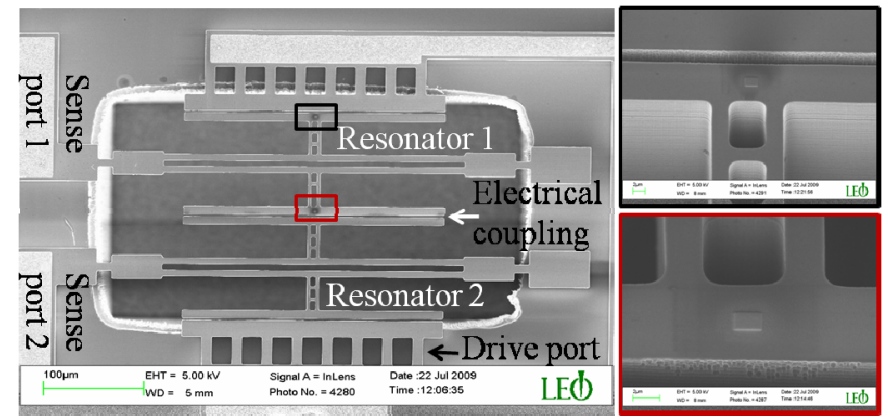

Figure 4. SEM image of electrically coupled DETF resonators - The insets show magnified images of the deposited platinum masses on resonator 1.

Both devices were fabricated in a commercial foundry process using the silicon-on-insulator microelectromechanical systems (SOI-MEMS) process through MEMSCAP Inc., USA.

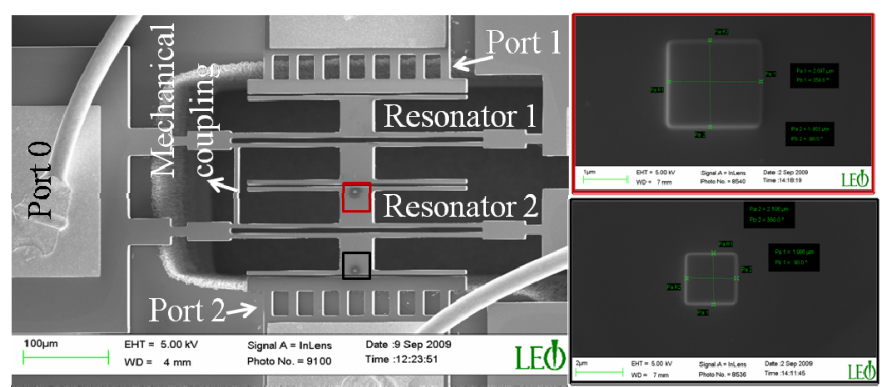

Figure 5. SEM image of mechanically coupled DETF resonators - the insets show magnified images of the deposited platinum masses on resonator 2 .

Each of the tines in both configurations was designed to be 25 $\mu \mathrm{m}$ thick, $300 \mu \mathrm{m}$ long and $6 \mu \mathrm{m}$ wide with a gap of $6 \mu \mathrm{m}$ between the tines. The resonators were driven and sensed using capacitive transduction with the drive and coupling gaps designed to be $2 \mu \mathrm{m}$ wide. Actuation was achieved using parallel plates of equal dimensions $(260 \mu \mathrm{m}$ long, $6 \mu \mathrm{m}$ wide, $25 \mu \mathrm{m}$ thick), attached to either side of each resonator in both configurations as illustrated in the Fig. 4 and 5. The open-loop transmission frequency responses were measured using a Vector Network analyzer (VNA) - Agilent 4396B. The resonators were actuated in the 'tuning fork' mode as the outof-phase motion of the resonators in this mode serves to cancel out the stresses at the anchors thereby enhancing the quality factor (Q) of resonance. The fabricated devices were tested under partial vacuum (50mTorr) in a custom vacuum chamber.

\section{B. Mechanically coupled DETF resonators}

Initially, in order to obtain an accurate estimate of the coupling spring constant, the loci of the two eigenvalues measured from the mechanically coupled DETF configuration were plotted as a function of stiffness variations induced on resonator 2 as illustrated in Fig. 6. This was achieved by applying a DC bias voltage of $10 \mathrm{~V}$ on port 0 (refer Fig. 5) while providing an $\mathrm{AC}$ input from the VNA corresponding to $-25 \mathrm{dBm}$ on port 1 . The $\mathrm{DC}$ voltage on resonator 1 was maintained at $0 \mathrm{~V}$ thereby resulting in a single port input drive force applied on resonator 1 . The DC bias voltage on port 2 was then tuned to alter the stiffness of resonator 2 maintaining that of resonator 1 constant as shown in Fig. 6.

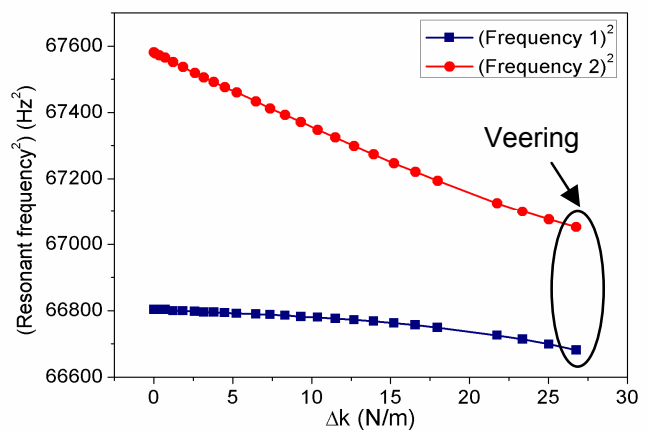

Figure 6. Veering of the loci of the eigenvalues observed when electrically varying the stiffness of resonator 2 .

It may be observed that as the loci of the two eigenvalues approach each other, the eigenvalues veer as predicted by (2). The eigenfunctions at this point of veering hence correspond to those represented in (4) and may be used to directly deduce the scaled coupling factor of the system. The value of $\kappa=k_{c} / k_{1}$ thus estimated corresponded to .0027776 . Deducing the value of $\kappa$ without performing the veering test, however, yielded a value of .00582036 - a value nearly 2 times higher. This directly translates to an enhancement in accuracy by nearly $200 \%$ (from (5)). After estimating the value of $\kappa$, keeping the voltage on port 0 at $10 \mathrm{~V}$, the ac power input was switched from port 1 to port 0 . A DC input voltage of $0 \mathrm{~V}$ was then applied on both ports 1 and 2. This allowed for the same force of actuation to be applied to both resonators thereby enabling us to directly deduce the eigenstates of the system by simply measuring the relative S21 transmission responses arising from the motional currents of resonators 1 and 2 generated at ports 1 and 2 respectively. As long as the modes do not overlap, each eigenstate is simply the normalized vector formed by the amplitudes thus measured. After measuring the initial eigenstates using this approach, two platinum patches (measuring approx. $2 \mu \mathrm{m} \times 2 \mu \mathrm{m} \times 300 \mathrm{~nm}$ and corresponding to a volumetric mass of approx. $51.5 \mathrm{pg}$ ), were deposited on the anti-nodal parallel plates of one of the mechanically coupled tuning forks (resonator 2) using the focused ion beam (FIB) (Fig. 5). The relative transmission responses of resonators 1 and 2 before and after mass addition (after cancelling the effects of capacitive feed-through current) are shown in Fig. 7. Comparing the responses, it may be observed that the relative shifts in the eigenstates correspond to $0.89 \%$ at first eigenvalue and $1.24 \%$ at the second. Based on the estimate of the scaled coupling factor derived from the veering test, the predicted variation corresponded to $1.05 \%$. Comparing the relative shifts in the eigenstates with those in resonant frequency (which corresponded to $0.0086 \%$ ), it can be observed that the variations in the eigenstates were nearly two 
orders of magnitude greater than corresponding shifts in the resonant frequency for the same perturbation in mass.

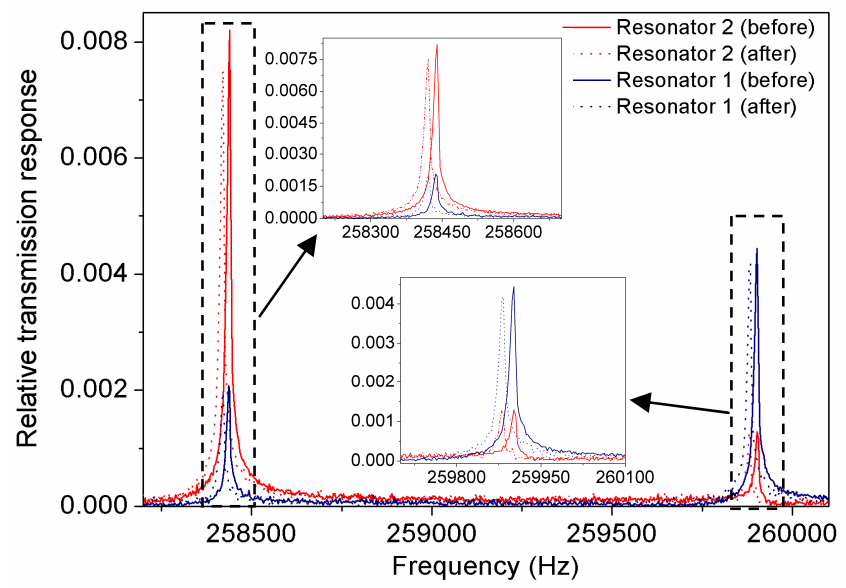

Figure 7. Experimentally observed relative $\mathrm{S} 21$ transmission responses measured from ports 1 and 2, before and after mass addition.

\section{Electrically coupled DETF resonators}

Unlike mechanical coupling, electrical coupling provides an added advantage in such a sensing paradigm as it can yield significantly weaker coupling spring constants, and consequently stronger localization. Furthermore, as the coupling spring is voltage tunable, it enables the realization of mode localized sensors with electrically tunable sensitivity (from (5) and (6)). In order to test the effects of spring tuning on parametric sensitivity of electrically coupled resonators utilizing this paradigm of sensing, the electrically coupled DETF configuration described in section III - A and illustrated in Fig. 4 was utilized. The initial eigenstates of the coupled resonators under varying effective coupling spring constants were characterized by applying DC bias voltages of equal magnitudes but opposite polarities on the two resonators and measuring the corresponding relative transmission responses. After measuring the initial eigenstates, two platinum patches (measuring approx. $1.8 \mu \mathrm{m} \times 1.8 \mu \mathrm{m} \times 180 \mathrm{~nm}$ and corresponding to a mass of approx. $25 \mathrm{pg}$ ), were deposited on the anti-nodal parallel plates of resonator 1 using the FIB (Fig. 4). The relative transmission responses of resonators 1 and 2 before and after mass addition for an applied bias of $+5 \mathrm{~V}$ and $-5 \mathrm{~V}$ respectively are shown in Fig. 8 . It can be noticed that the relative shifts in the eigenstates for the induced mass addition correspond to $1.27 \%$ and $1.88 \%$ at the two eigenvalues. Comparing these with relative shifts in resonant frequency $(\cong 0.002 \%)$, the variations in the eigenstates were found to be nearly three orders of magnitude greater than corresponding resonant frequency shifts for the same perturbation in mass. Owing to significantly weaker coupling spring constants, the relative shifts in the eigenstates are higher than those observed in the mechanically coupled configuration for lower induced mass shift. Furthermore, measured relative shifts in the eigenstates for the same induced mass additions were observed to increase with lower $k_{c}$ as illustrated in Fig. 9, indicating that tuning $k_{c}$ to lower values result in stronger localization of the vibration modes as predicted by (5). Tunability in parametric sensitivity of nearly $400 \%$ was observed by tuning the electrical coupling spring.

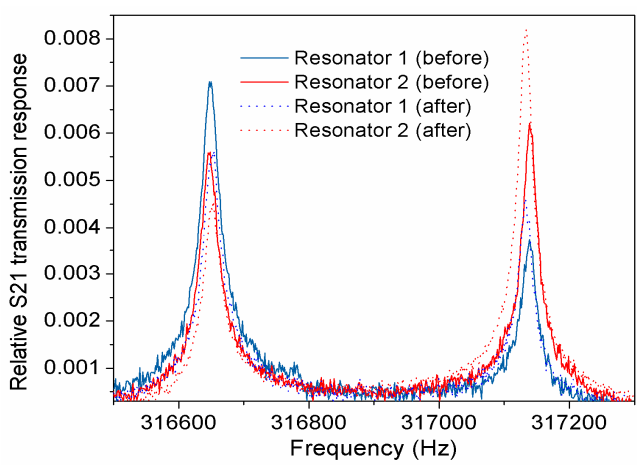

Figure 8. Experimentally observed relative $\mathrm{S} 21$ transmission responses measured from resonators 1 and 2, before and after mass addition.

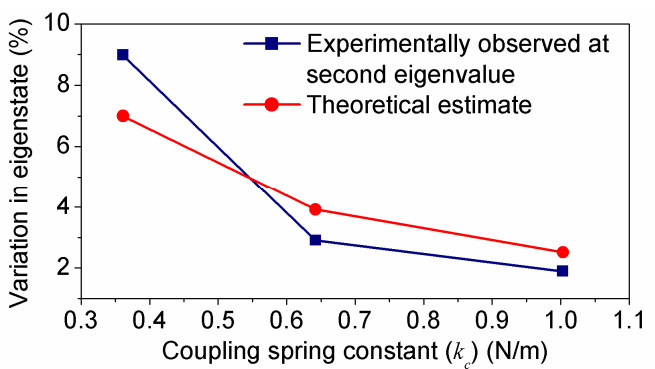

Figure 9. Variation in eigenstate for the same induced perturbation in mass at varying effective coupling spring constants.

\section{CONCLUSIONS}

This paper compares the effects of electrical and mechanical coupling on the parametric sensitivity of mode localized mass sensors. The phenomenon of eigenvalue curve veering is employed to accurately quantify the strength of internal coupling in the mechanically coupled configuration, improving the accuracy of the predicted shifts in eigenstates by over a factor of two. The tunability of the coupling spring constant in electrically coupled mode-localized sensors is also utilized to realize a mass sensor with tunable sensitivity.

\section{REFERENCES}

[1] M. Spletzer, A. Raman, A. Q. Wu, X. Xu, and R. Reifenberger, "Ultrasenstive mass sensing using mode localization in coupled microcantilevers," Appl. Phys. Lett., vol. 88, pp. 254102, 2006.

[2] M. Spletzer, A. Raman, H. Sumali, and J. P. Sullivan, "Highly sensitive mass detection and identification using vibration localization in coupled microcantilever arrays," Appl. Phys. Lett., vol. 92, pp. 114102, 2008.

[3] P. Thiruvenkatanathan, J. Yan, J. Woodhouse, and A. A. Seshia, "Enhancing parametric sensitivity in electrically coupled MEMS resonators," J. Microlectromech. Syst., in press, 2009.

[4] P. Thiruvenkatanathan, J. Yan, and A. A. Seshia, "Common mode rejection in electrically coupled MEMS resonators utilizing mode localization for sensing applications," IEEE Freq. Cont. Symp.,France, April 21-24, 2009.

[5] C. Pierre, "Mode localization and eigenvalue loci veering phenomena in disordered structures," J. Sound Vib., vol. 126, pp. 485-502, 1988.

[6] X. L. Liu, "Behaviour of derivative of eigenvalues and eigenvectors in curve veering and mode localization and their relation to close eigenvalues," J. Sound Vib., vol. 256, pp. 551-564, 2002.

[7] A. W. Leissa, "On a curve veering aberration," J. App. Math. Phys. (ZAMP), vol. 25, pp. 99-111, 1974. 